\title{
In vivo photoacoustic imaging of major blood vessels in the pancreas and liver during surgery
}

Kelley M. Kempski

Alycen Wiacek

Michelle Graham

Eduardo González

Bria Goodson

Derek Allman

Jasmin Palmer

Huayu Hou

Sarah Beck

Jin $\mathrm{He}$

Muyinatu A. Lediju Bell 


\title{
In vivo photoacoustic imaging of major blood vessels in the pancreas and liver during surgery
}

\author{
Kelley M. Kempski, ${ }^{a}$ Alycen Wiacek, ${ }^{b}$ Michelle Graham,, ${ }^{b}$ Eduardo González, ${ }^{\mathrm{c}}$ Bria Goodson, ${ }^{\mathrm{d}}$ Derek Allman, \\ Jasmin Palmer, ${ }^{e}$ Huayu Hou,${ }^{b}$ Sarah Beck, ${ }^{\dagger}$ Jin He,,${ }^{g, h}$ and Muyinatu A. Lediju Bell ${ }^{b, c, i, *}$ \\ aUniversity of Delaware, Department of Biomedical Engineering, Newark, Delaware, United States \\ bJohns Hopkins University, Department of Electrical and Computer Engineering, Baltimore, Maryland, United States \\ 'Johns Hopkins University, Department of Biomedical Engineering, Baltimore, Maryland, United States \\ ${ }^{\mathrm{d} D e l t a}$ State University, Department of Biology, Cleveland, Mississippi, United States \\ 'Massachusetts Institute of Technology, Department of Mechanical Engineering, Cambridge, Massachusetts, United States \\ fJohns Hopkins Medicine, Department of Molecular and Comparative Pathobiology, Baltimore, Maryland, United States \\ gJohns Hopkins Medicine, Department of Surgery, Baltimore, Maryland, United States \\ hJohns Hopkins Medicine, Department of Oncology, Baltimore, Maryland, United States \\ iJohns Hopkins University, Department of Computer Science, Baltimore, Maryland, United States
}

\begin{abstract}
Abdominal surgeries carry considerable risk of gastrointestinal and intra-abdominal hemorrhage, which could possibly cause patient death. Photoacoustic imaging is one solution to overcome this challenge by providing visualization of major blood vessels during surgery. We investigate the feasibility of in vivo blood vessel visualization for photoacoustic-guided liver and pancreas surgeries. In vivo photoacoustic imaging of major blood vessels in these two abdominal organs was successfully achieved after a laparotomy was performed on two swine. Three-dimensional photoacoustic imaging with a robot-controlled ultrasound (US) probe and color Doppler imaging were used to confirm vessel locations. Blood vessels in the in vivo liver were visualized with energies of 20 to $40 \mathrm{~mJ}$, resulting in 10 to $15 \mathrm{~dB}$ vessel contrast. Similarly, an energy of $36 \mathrm{~mJ}$ was sufficient to visualize vessels in the pancreas with up to $17.3 \mathrm{~dB}$ contrast. We observed that photoacoustic signals were more focused when the light source encountered a major vessel in the liver. This observation can be used to distinguish major blood vessels in the image plane from the more diffuse signals associated with smaller blood vessels in the surrounding tissue. A postsurgery histopathological analysis was performed on resected pancreatic and liver tissues to explore possible laser-related damage. Results are generally promising for photoacoustic-guided abdominal surgery when the US probe is fixed and the light source is used to interrogate the surgical workspace. These findings are additionally applicable to other procedures that may benefit from photoacoustic-guided interventional imaging of the liver and pancreas (e.g., biopsy and guidance of radiofrequency ablation lesions in the liver). () The Authors. Published by SPIE under a Creative Commons Attribution 4.0 Unported License. Distribution or reproduction of this work in whole or in part requires full attribution of the original publication, including its DOI. [DOI: 10.1117/1.JBO.24.12.121905]

Keywords: photoacoustic-guided surgery; interventional imaging; liver surgery; pancreatic surgery.
\end{abstract}

Paper 190094SSR received Mar. 31, 2019; accepted for publication Jul. 22, 2019; published online Aug. 13 , 2019.

\section{Introduction}

Several techniques and tools exist to prevent excessive bleeding during pancreatic and liver surgeries, such as lowering central venous pressure ${ }^{1,2}$ and various clamping methods. ${ }^{2,3}$ In addition, devices such as the harmonic scalpel and the cavitron ultrasonic surgical aspirator ${ }^{3,4}$ control bleeding by emitting heat through vibrating at an ultrasonic frequency to coagulate the tissue and oscillating to cause explosion of cells with high water content, respectively. Despite these modern surgical advances, there is still a high risk of gastrointestinal and intra-abdominal hemorrhage and possibly death. ${ }^{3,5,6}$ Postoperative morbidity occurs in $23 \%$ to $46 \%$ of patients who hemorrhage during liver resections, and death occurs in $4 \%$ to $5 \%$ of these patients. ${ }^{3}$ Similarly, hemorrhaging occurs in $1 \%$ to $8 \%$ of all pancreatic resections and $2 \%$ to $18 \%$ of pancreaticoduodenectomies, in addition to causing $11 \%$ to $38 \%$ of postoperative morbidity for pancreatic resections and pancreaticoduodenectomies. ${ }^{5,6}$ Thus, there is a clinical need to distinguish major blood vessels during surgery to reduce the risk of arterial and venous injuries.

*Address all correspondence to Muyinatu A. Lediju Bell, E-mail: mledijubell@ jhu.edu
Preventive measures can be taken to reduce hemorrhaging by visualizing major blood vessels to mitigate the risk of accidentally cutting these blood vessels while operating. Ultrasound (US)-based imaging methods are among the most promising techniques for real-time visualization during surgery, due to the low cost, portability, and real-time, multiplanar information that is typically available with US-based techniques. For example, color Doppler imaging is one option to determine the location of blood vessels during surgery, as this US-based imaging mode provides information about the presence of blood flow. However, Doppler imaging is limited to providing a map of flow throughout the US plane, with conventional US imaging required to determine where this flow is located in relation to surgical tools that appear in the US plane. ${ }^{7}$ Relying solely on conventional US imaging to visualize surgical tools is problematic because the metallic surgical tools tend to cause reverberation artifacts that make the tools difficult to locate. ${ }^{8}$ In addition, the presence of acoustic clutter ${ }^{9}$ confounds surgical tool localization with conventional US imaging, particularly in obese patients. ${ }^{10}$ Photoacoustic images are robust to these particular long-standing challenges with US imaging, as demonstrated in our previous publication. ${ }^{11}$ Therefore, photoacoustic imaging has the potential to produce superior information about blood 
vessel locations relative to surgical tool tip locations, when compared to Doppler imaging combined with US imaging.

Photoacoustic imaging is implemented by irradiating various targets using a laser, which produces acoustic pressure that is recorded by an US probe. ${ }^{12-14}$ Photoacoustic imaging can be paired with traditional US imaging to improve specificity, sensitivity, and visualization of tissue boundaries, which may allow a clinician to better distinguish between a blood vessel and soft tissue. ${ }^{13,15,16}$ For example, this technique has successfully distinguished blood vessels in mouse brains. ${ }^{17}$ In addition, the high optical absorption of metal ${ }^{18}$ enables both a tool tip and a nearby major blood vessel to be simultaneously visualized in the photoacoustic image plane. ${ }^{19}$ One additional benefit of photoacoustic imaging is that it does not use harmful ionizing radiation.

The possibility of using real-time photoacoustic images to guide surgeries is a relatively new area of investigation. As photoacoustic imaging has excellent capabilities to visualize blood vessels, one recent concept of photoacoustic-guided surgery is centered on visualizing major blood vessels during surgery to avoid significant blood loss and accidental injury to these major vessels, which would be a significant step toward reducing the risk of patient death during surgery. The feasibility of this concept was previously demonstrated for potential guidance in neurosurgeries,${ }^{20}$ hysterectomies, ${ }^{21,22}$ spinal fusion surgeries, ${ }^{23}$ and fetal surgeries. ${ }^{24}$ To achieve this new paradigm for surgical guidance, specialized light delivery systems can surround existing surgical tools to optically excite the surgical field, and the US probe may be placed externally to receive the resulting photoacoustic images, as previously demonstrated for a neurosurgical drill bit ${ }^{19}$ and a scissor tool for minimally invasive surgery. ${ }^{21}$ This concept of interventional photoacoustic imaging may also be integrated with robots for minimally invasive surgery, such as the da Vinci teleoperated robot, ${ }^{21,22,25-28}$ or to enable photoacoustic-based visual servoing. ${ }^{11,29}$

The potential for real-time photoacoustic liver imaging was previously investigated using phantoms, ${ }^{30-32}$ with promising applications to photoacoustic-guided liver surgery. One major challenge is that visualizing blood vessels in the liver is particularly difficult because the absorption spectrum of the liver tissue is similar to that of blood, considering that the liver contains many small and large vessels. ${ }^{30,33}$ Despite this known challenge, Bell et al. ${ }^{34}$ achieved photoacoustic signals at depths of $3 \mathrm{~cm}$ with $8 \mathrm{~mJ}$ of energy emitted from an interstitial light source inserted in an ex vivo liver tissue containing retained blood.
To achieve these images, light from a 1064-nm Nd:YAG laser was delivered through a 1-mm core diameter optical fiber surrounded by a light diffusing sheath. ${ }^{34}$ Similarly, Mitcham et al. ${ }^{31}$ achieved photoacoustic signals as deep as $7 \mathrm{~cm}$ with an interstitial light source inserted in the ex vivo liver tissue. No visible tissue damage was caused by the 8-mJ Nd:YAG laser energy that was applied with a $1-\mathrm{mm}$ core diameter fiber. ${ }^{31}$

The lack of tissue damage in the ex vivo liver tissue is an important observation, considering that the fluence values were as high as $30 \mathrm{~mJ} / \mathrm{cm}^{2}$, which exceeds the laser safety limit of $22 \mathrm{~mJ} / \mathrm{cm}^{2}$ for skin at a wavelength of $720 \mathrm{~nm}$. However, skin has higher optical scattering than liver ${ }^{31}$ and pancreatic tissues, as reported in Table 1 . Thus, these and other internal abdominal tissues can potentially withstand greater values of energy and fluence than those currently defined with the laser safety limits for skin. When compared to the two previous studies, ${ }^{31,34}$ Hill et al. ${ }^{30}$ applied the greatest laser energies (up to $50 \mathrm{~mJ}$ ) to obtain photoacoustic images of ex vivo porcine liver tissue, where the different lobes were visualized at depths exceeding $2 \mathrm{~cm}$ with a 1064-nm Nd:YAG laser and a 910- $\mu$ m diameter fiber.

To the authors' knowledge, no previous studies investigated the feasibility of interventional photoacoustic liver imaging in vivo; thus, expectations regarding the performance of this imaging method during liver and other abdominal surgeries remain to be determined. The pig abdominal vasculature, particularly vessels surrounding the pancreas and liver, is similar to that of humans, including similarities in overall size, diameter, wall thickness, lumen area, cross-sectional area, vascular density, structure, and nomenclature. ${ }^{45-49}$ One exception is that pigs only have a single inferior pancreaticoduodenal artery, whereas humans have an anterior and posterior inferior pancreaticoduodenal artery. ${ }^{49}$ In addition, the pig and human liver and pancreatic tissues have similar structure, as well as similar optical and thermal properties to those of human tissues, as detailed in Table 1 . Thus, the pig is a suitable animal model to study the feasibility of in vivo photoacoustic vessel visualization for human liver and pancreas surgeries.

This paper expands our associated conference paper ${ }^{50}$ to explore in vivo photoacoustic imaging of major pancreatic and hepatic vessels in a pig animal model. Section 2 describes our surgical procedure as well as ex vivo experiments that were performed to confirm the results seen during the in vivo procedure. Section 3 presents the results of our study including photoacoustic images from the in vivo pancreas and both in vivo

Table 1 Comparison of optical properties and thermal conductivity at $37^{\circ} \mathrm{C}$ in human and pig tissues.

\begin{tabular}{|c|c|c|c|c|c|}
\hline Wavelength (nm) & Tissue & Absorption $\left(\mathrm{mm}^{-1}\right)$ & Scattering $\left(\mathrm{mm}^{-1}\right)$ & $\begin{array}{l}\text { Anisotropy } \\
\text { factor, } g\end{array}$ & $\begin{array}{l}\text { Thermal conductivity } \\
\left(\mathrm{Wm}^{-1} \mathrm{~K}^{-1}\right)\end{array}$ \\
\hline \multirow[t]{3}{*}{750} & Skin (human) ${ }^{35,36}$ & 0.5 & 100 & - & 0.24 \\
\hline & Liver (pig) $)^{37-39}$ & 0.1 & 7 & 0.9 to 1 & 0.53 \\
\hline & Liver (human) ${ }^{38-41}$ & $<0.1$ & -10 & - & 0.53 \\
\hline \multirow[t]{2}{*}{1060} & Liver (pig) $)^{37,39,42}$ & 1 & -5 & 0.9 to 1 & 0.53 \\
\hline & Liver (human) ${ }^{37-41,43}$ & $<0.1$ to 0.6 & 5 & 0.9 to 1 & 0.53 \\
\hline 1064 & Pancreas (pig) ${ }^{44}$ & 0.02 to 0.03 & 0.025 & - & 0.54 \\
\hline
\end{tabular}

aThermal conductivity does not depend on wavelength. 
and ex vivo liver tissue. In addition, we present the pathology associated with using a laser to image the in vivo liver and pancreatic tissue. Section 4 discusses the strengths and challenges of a photoacoustic approach to surgical guidance in the abdomen, as well as a plan for future studies to further investigate these challenges. Finally, we conclude our paper in Sec. 5.

\section{Methods}

\subsection{Experimental Setup}

All procedures were approved by the Johns Hopkins University Institutional Animal Care and Use Committee and all experiments were conducted at the Johns Hopkins Minimally Invasive Surgical Training and Innovation Center. Two Sus domesticus crossbred domestic pigs (5 and 6 months, 65 and 90 lbs., both female) were obtained for this study. Animals were treated humanely. Pigs were anesthetized and subsequently intubated to provide gas anesthesia throughout the surgery. Respiration was controlled with a ventilator that supplied medical air. A midline incision exposed the abdominal organs. Blood pressure and heart rate were monitored throughout the surgery and imaging procedures.

The photoacoustic imaging system, which was placed next to the operating table, as shown in Fig. 1, consisted of an Alpinion ECUBE 12R US system (Seoul, Korea) synchronized with a Phocus Mobile laser (OPOTEK, Carlsbad, California). The US system was connected to a 128-element Alpinion L3-8 linear array transducer, which has a center frequency of $5 \mathrm{MHz}$, emitted an US transmit focus of $3 \mathrm{~cm}$, and contained an active receive aperture limited to 64 elements. The laser was connected to a 5-mm-diameter fiber bundle. Although we also connected the laser to a 1-mm-diameter optical fiber and attempted to image blood vessels in the pancreas with this fiber, we were unsuccessful. Thus, all images in this paper show our success with the 5-mm-diameter fiber bundle.

A fixed laser wavelength of $750 \mathrm{~nm}$ was chosen to visualize blood vessels throughout the experiments. The wavelength was fixed, given our primary goal of visualizing (rather than characterizing) major blood vessels during surgery and our reasoning that one wavelength is sufficient to inform the surgeon of the presence or absence of a blood vessel during surgery. The US transducer was either held by a Sawyer robot (Rethink Robotics, Boston, Massachusetts) or by hand. In addition to acquiring photoacoustic images, the US system was also used to acquire coregistered US images and color Doppler images to confirm vessel locations.

\subsection{Surgical Procedure}

A laparotomy was performed to gain access to the liver and pancreas, and photoacoustic imaging was used to identify abdominal blood vessels in and around the pancreas and liver. To mimic a surgical scenario where the light source would surround a surgical tool and the US probe would be relatively stationary, while the surgical tool is operated, ${ }^{19,21,22,25,51}$ the fiber bundle was held at a variable angle relative to the transducer. Given this motivation and the existing literature describing that the maximum photoacoustic signal is achieved when the light axis intersects both the photoacoustic imaging target and the imaging plane, ${ }^{30,52}$ we prioritized flexibility of the fiber bundle axis relative to the orientation of the imaging plane. Building on knowledge obtained from controlled measurements of light delivery placement relative to the imaging plane and a target of interest, ${ }^{30,52-55}$ photoacoustic images were optimized by manipulating the light source to maximize the amplitude and appearance of photoacoustic signals, similar to the free-hand optimization procedure that a surgeon would perform with a specialized light delivery system attached to the surgical tool.

When imaging the pancreas, the superior mesenteric vein (SMV), right gastroepiploic vein (RGEV), and right gastroepiploic artery (RGEA) were visualized. These vessels are annotated in the schematic diagram of Fig. 2(a). The section of pancreatic tissue exposed to the laser was resected. In addition, pancreatic tissue that was not exposed to laser light was resected as a control specimen. The energies used to image the pancreas with the 1- and 5-mm-diameter light sources are shown in Fig. 3(a) (top).

The hepatic veins [see Fig. 2(b)] were visualized when imaging the liver. The range of energies used to image the pancreas with the 5-mm-diameter fiber bundle is shown in Fig. 3(b) (top). Energies of 20 to $40 \mathrm{~mJ}$ in 5-mJ increments were used to excite

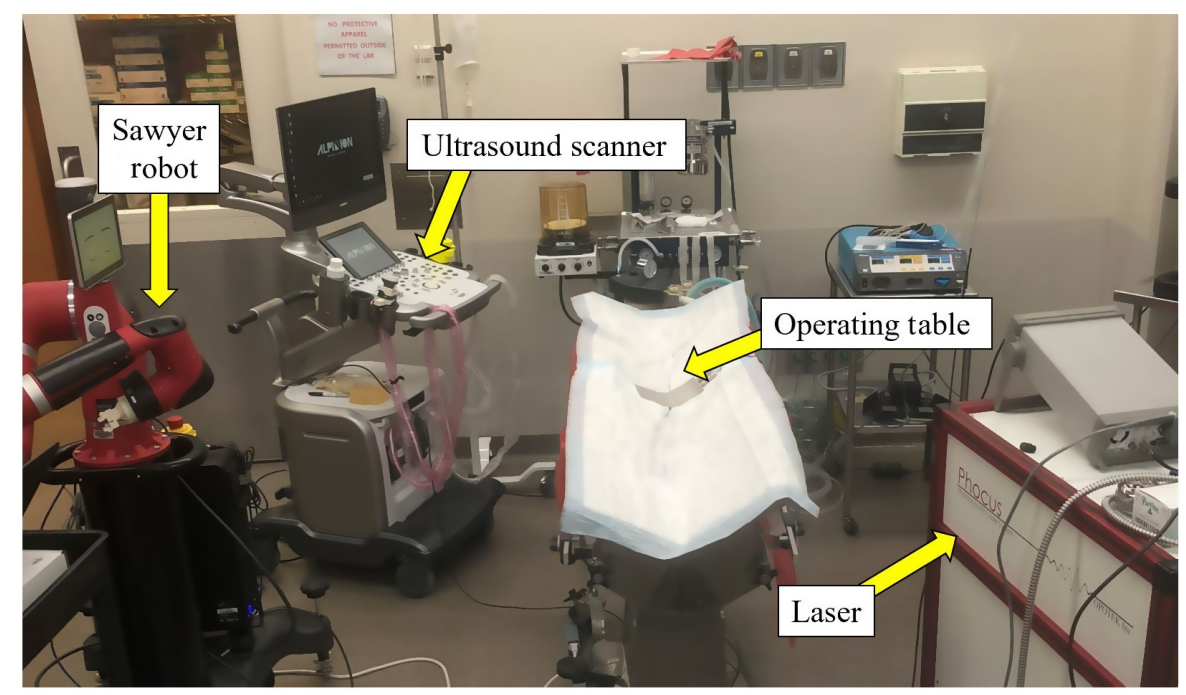

Fig. 1 Imaging equipment and surgical environment for in vivo porcine laparotomy. 
(a)

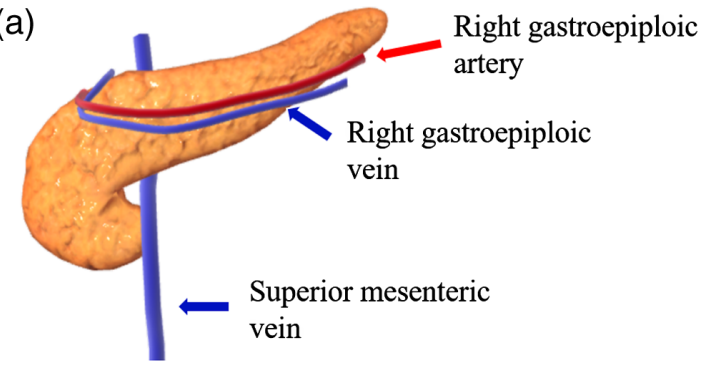

(b)

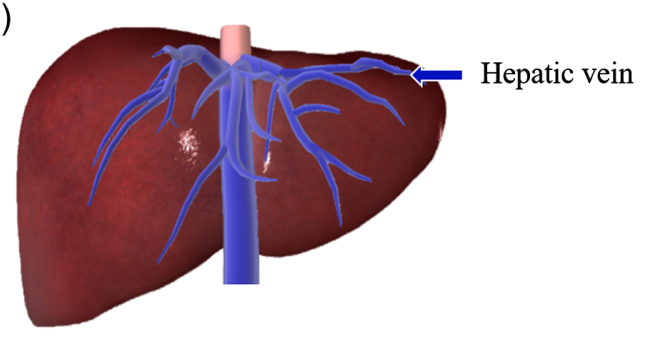

Fig. 2 Vessels imaged in the (a) pancreas and (b) liver. The hepatic veins are inside of the liver but are displayed overlaid on the liver to allow for visualization of the vessel locations. ${ }^{56}$

the blood vessels to determine the minimum required energy needed to visualize the liver vasculature. A three-dimensional (3-D) reconstruction of the hepatic vein was created with the robot-held US probe translating in 1-mm increments across the liver surface, pausing at each increment to acquire end-inspiration photoacoustic or color Doppler images. The liver tissue exposed to the laser was resected as well as a control from a portion of the same liver lobe that was not in direct contact with the 5-mm-diameter fiber bundle. A control from the liver was additionally resected prior to any imaging. The resected pancreas and liver tissues underwent histopathological analysis with hematoxylin and eosin staining by coauthor S.B., a boardcertified veterinary pathologist.

\subsection{Energy Measurements and Fluence Calculations}

Energy was manually adjusted throughout the procedure. The input energy into the optical fiber or fiber bundle was recorded with a power meter internal to the laser system. These input energies were calibrated to output energies (recorded with a second external power meter) using the average of both pre- and post-experiment input-to-output energy measurements. The recorded energy as a function of time to image the pancreas and the liver prior to tissue resection is shown at the top of Figs. 3(a) and $3(\mathrm{~b})$, respectively. Fluence, $\Phi$, was calculated from these recorded energies, $E$, and the area of the incident light, which is defined as

$\Phi=\frac{E}{\pi r^{2}}$,

where $r$ represents the radius of the 5-mm-diameter fiber bundle or the 1-mm-diameter fiber bundle, assuming that each light source was in direct contact with the tissue. The calculated fluence used for imaging the in vivo pancreas and liver is shown at the bottom of Figs. 3(a) and 3(b), respectively, as a function of time. Note that these calculations represent a conservative estimate of fluence, as the area of incident light could be larger than the diameter used for the calculations, due to light scattering on the tissue surface (as observed in recorded photographs) and possibly due to divergence of the light if the laser was not in direct contact with the tissue as intended.

\subsection{Ex Vivo Experiment}

An ex vivo experiment was performed to investigate the in vivo observations of potential out-of-plane vessels. The ex vivo phantom consisted of bovine liver tissue that surrounded tubes filled with human blood. The three parallel blood vessels were set up to mimic the RGEV, SMV, and RGEA. A perpendicular blood vessel was used to mimic the out-of-plane vessel, as illustrated

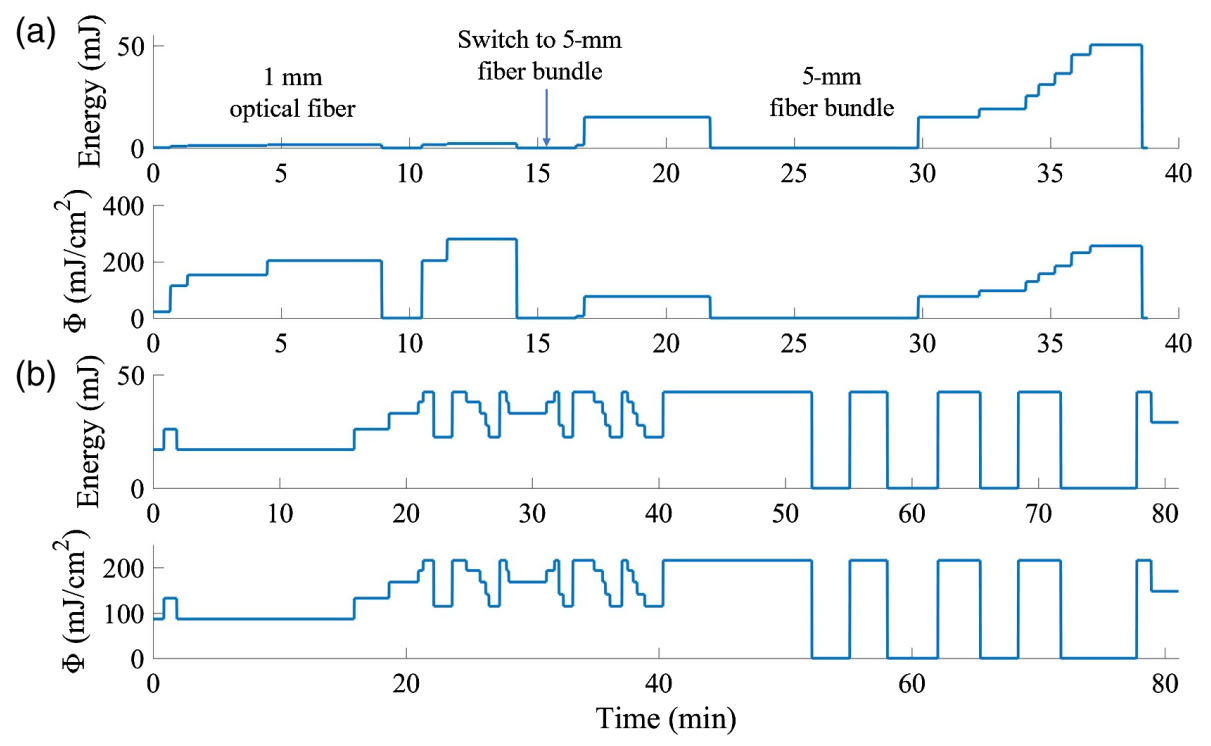

Fig. 3 Energy versus time graphs and fluence $(\Phi)$ versus time graphs for imaging the (a) pancreas and (b) liver. The 1-and 5-mm-diameter fiber bundles were used to image the pancreas during the time points indicated. The 5-mm-diameter fiber bundle was used to image the liver. The methods implemented to measure energy and calculate fluence are detailed in Sec. 2.3. 

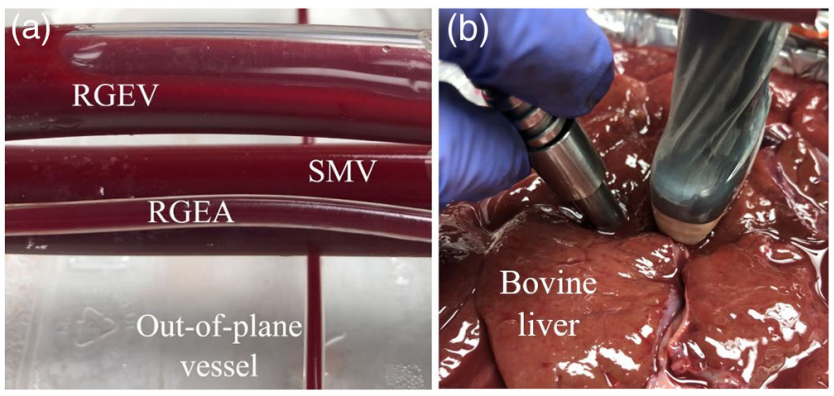

Fig. 4 Photographs of the ex vivo experiment setup. (a) Plastic tubes were filled with human blood to mimic the RGEV, RGEA, SMV, and the out-of-plane blood vessel. (b) Bovine liver tissue surrounded the phantom blood vessels while the L3-8 linear transducer and 5-mmdiameter fiber bundle were used to visualize the blood vessels.

in Fig. 4. Energies of 5.5 to $70.1 \mathrm{~mJ}$ were used to visualize blood in this ex vivo experiment.

\subsection{Image Display Parameters and Data Analysis}

Photoacoustic images were created using delay-and-sum (DAS) beamforming and short-lag spatial coherence (SLSC) beamforming. ${ }^{34,57}$ SLSC images were generated with the short-lag value equal to one (or $2 \%$ of the receive aperture) and the axial correlation kernel equal to one wavelength. These two SLSC image parameters are defined as $M$ and $k$, respectively, in previous publications. ${ }^{34,57}$ Images were normalized to the brightest pixel, log-compressed, and a minimum threshold value was selected to optimize signal display.

Contrast was calculated using beamformed data prior to normalization and log compression, according to the following equation:

Contrast $=20 \log _{10}\left(\frac{\mu_{\text {vessel }}}{\mu_{\text {background }}}\right)$,

where $\mu_{\text {vessel }}$ and $\mu_{\text {background }}$ are the mean signals within a region of interest (ROI) inside of a blood vessel and in the background, respectively. The ROI was created by outlining the borders of the signal inside of the blood vessel and creating an ROI of the same size and at the same depth in the background. ROIs were $4 \mathrm{~mm}$ in the lateral direction and $2 \mathrm{~mm}$ in the axial direction.

\section{Results}

\subsection{In Vivo Pancreas Imaging}

Figure 5 shows one result from the in vivo pancreas study. The US image with photoacoustic overlay is shown in Fig. 5(a). These images were created with DAS beamforming. The same photoacoustic channel data were beamformed using the SLSC approach, as shown in Fig. 5(b). This SLSC image is overlaid on the US image in Fig. 5(c). A Doppler image confirming the location of blood vessels in the pancreas is shown in Fig. 5(d).

Throughout the respiratory cycle, the pancreas moved in a relatively predictable motion due to the mechanical ventilator that was used to maintain respiration. However, the transducer and fiber source were fixed (i.e., they were not moving with the respiratory cycle). Thus, any observed signal motion over time indicates movement due to respiration. Contrast was measured to assess this type of motion. Figure 5(e) shows the measured contrast of the three signals in Fig. 5(b) as a function of time for image frames acquired during multiple respiratory cycles.

We observed three distinct coherent signals in the in vivo photoacoustic images of the pancreas [labeled as 1,2, and 3 in Fig. 5(b)]. Each signal consistently appeared and disappeared with respiration, as shown in Fig. 5(e). The source of these signals could be blood vessels (either in the imaging plane or outside of the imaging plane), reflection artifacts, or signals from the location of the light source due to the higher fluence where the light source is incident on the tissue, as seen in previous experiments with interstitial light sources. ${ }^{34}$ The color Doppler image in Fig. 5(d) shows that there is blood flow in the RGEV, RGEA, and SMV, which indicates that signal \#2 could be associated with blood, although photoacoustic signals are not present in the entire RGEV.

The reason for the partial display of the signal is unknown, but one possible explanation could be locally higher absorption due to the location of a valve in the vessel. Due to this uncertainty, it could be argued that signal \#2 did not originate from the blood in the RGEV and instead could be an artifact, particularly if signals \#1 and \#2 in the in vivo pancreas image of Fig. 5(a)
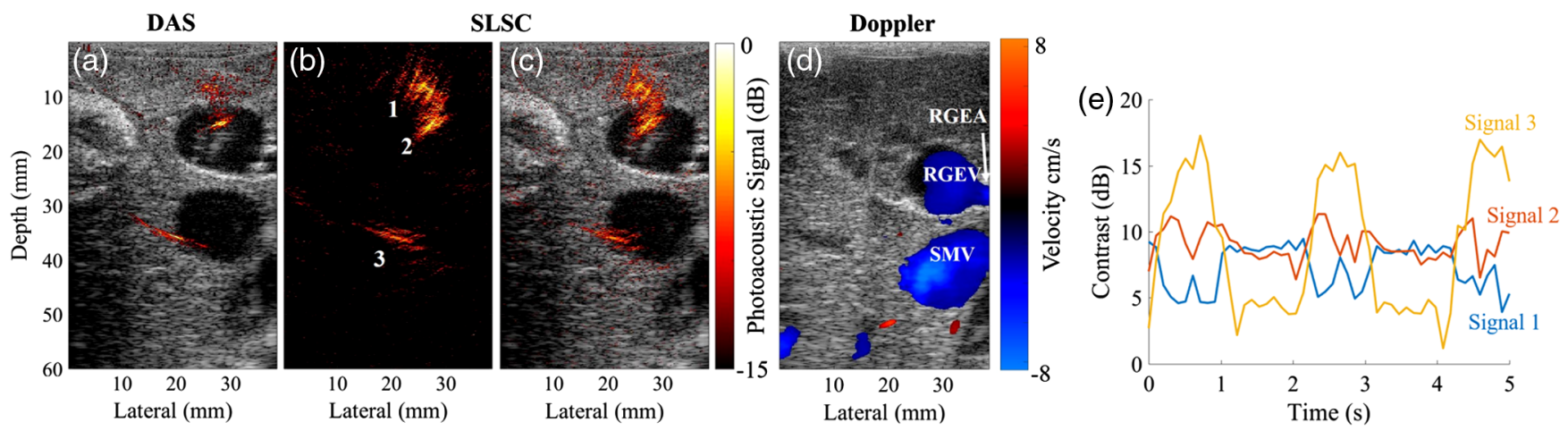

Fig. 5 (a) DAS beamformed photoacoustic image of signals in in vivo pancreas overlaid on coregistered US image. (b) Corresponding SLSC beamformed photoacoustic image created with the same channel data. (c) SLSC beamformed photoacoustic image overlaid on US image. (d) Color Doppler confirmation of blood flow in vessels. (e) Contrast of the three signals in DAS photoacoustic images. The photoacoustic images shown here and the photoacoustic images used for the contrast measurements were acquired with an energy of $36 \mathrm{~mJ}$. 
were not distinct from each other. However, because signals \#1 and \#2 are distinct in the DAS image and have distinctly different contrast measurements with changes in respiration, we hypothesize that signal \#2 originates from the blood.

The use of coherence-based SLSC beamforming supports our hypothesis that these signals originate from two independent coherent sources, because they appear as two distinct signals in the SLSC images. If there was a single source causing these signals, these signals would instead be merged together as one coherent source in the SLSC image. The SLSC image also confirms that coherent signals from the blood inside of the entire RGEV were not obtained.

While signal \#1 could be associated with the location of the light source, this is unlikely because the appearance of this signal changed with the respiratory cycle, while the US probe and fiber bundle were held stationary, as demonstrated in Fig. 5(e). If this signal were solely caused by the stationary light source primarily interacting with tissue, then its contrast would be more constant with changes in respiration. Another possible explanation is that this signal could be a reflection artifact. However, reflection artifacts typically appear deeper than true signals. Thus, we hypothesize that signal \#1 is most likely an out-ofplane blood vessel. Similarly, due to signal \#3 changing with respiration, as shown in Fig. 5(e), we hypothesize that this signal also originates from an out-of-plane blood vessel. These out-ofplane vessels can be resolved with 3-D imaging, as demonstrated in Sec. 3.4.

\subsection{Out-of-Plane Vessel Confirmation}

The ex vivo experiment described in Sec. 2.4 was performed to test our hypotheses about the source of the signals in the in vivo pancreas data. Although the fiber bundle was stationary while the tissue moved with respiration during the in vivo pancreas experiment, by inverting this process and keeping the ex vivo tissue stationary while moving the fiber bundle, we recreated our in vivo observations of photoacoustic signals appearing and disappearing from the image plane in this more controlled ex vivo experimental environment.

Figures 6(a)-6(c) show that the photoacoustic signal from the ex vivo out-of-plane vessel mimicked in vivo photoacoustic signal \#3 in Fig. 5(b). Figure 6(d) shows contrast as a function of time for this ex vivo experiment, and this result supports the hypothesis that signals \#1 and \#3 are associated with outof-plane blood vessels. In particular, the cyclic temporal appearance of the measured photoacoustic signal contrast in Fig. 6(d) is similar to that of the in vivo signals \#1 and \#3 in Fig. 5(e).
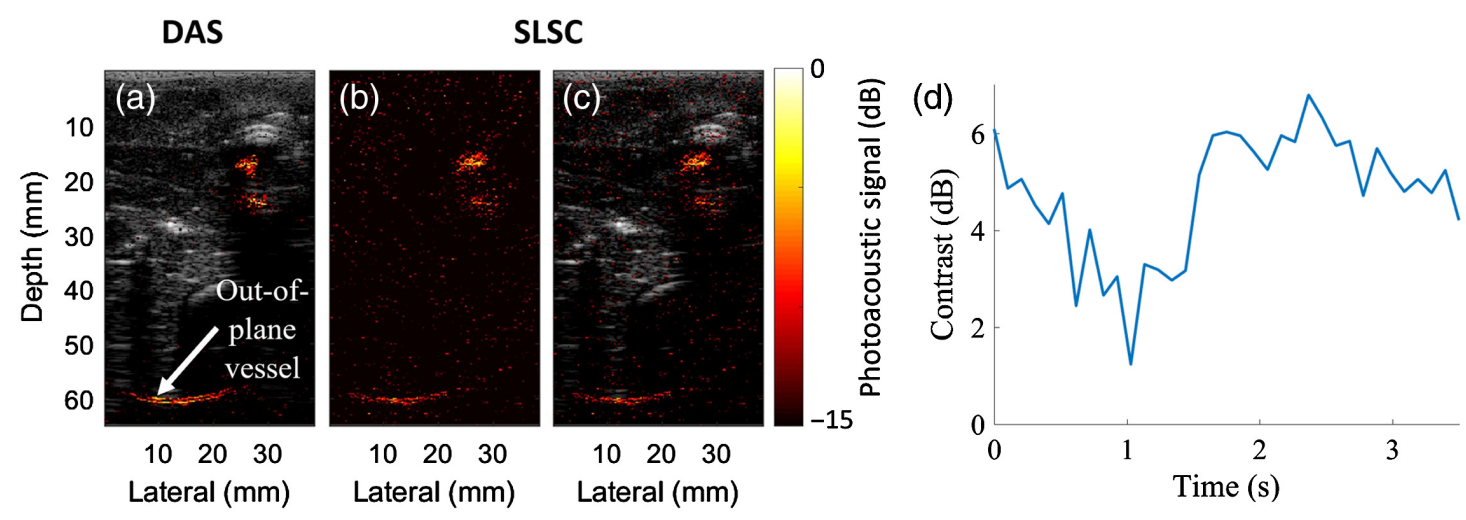

Fig. 6 (a) DAS beamformed photoacoustic image overlaid on US image of ex vivo blood vessels. (b) Corresponding SLSC beamformed photoacoustic image created with the same channel data. (c) SLSC beamformed photoacoustic image overlaid on US image. These photoacoustic images were acquired with an energy of $30.5 \mathrm{~mJ}$. (d) Contrast of the out-of-plane vessel signal, measured in the DAS photoacoustic image as the light source location was moved to mimic one respiratory cycle.
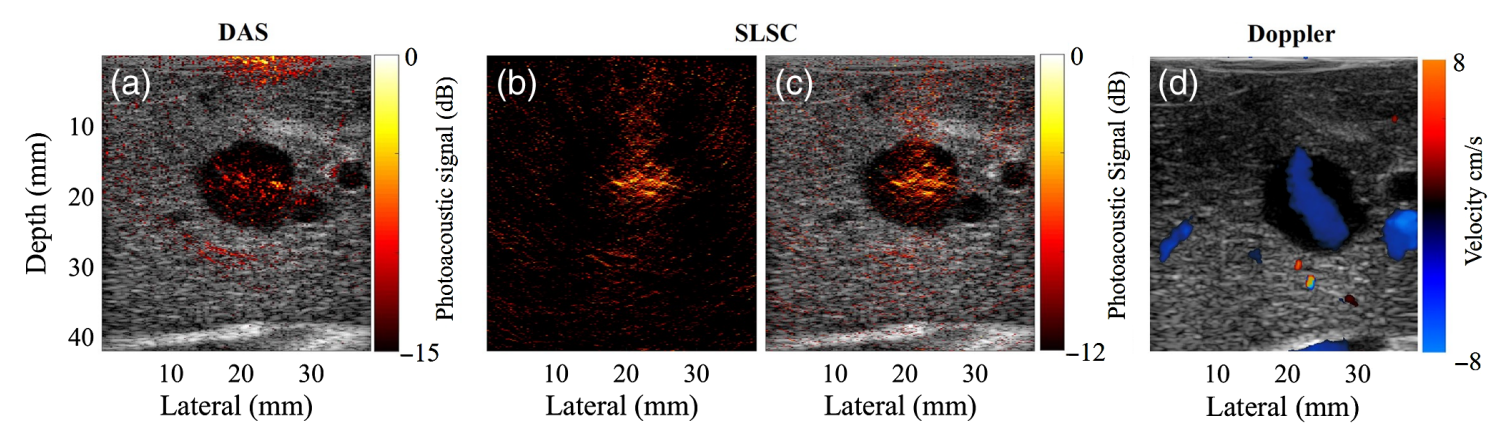

Fig. 7 (a) DAS beamformed photoacoustic image of signals in in vivo liver overlaid on coregistered US image. (b) Corresponding SLSC beamformed photoacoustic image created with the same channel data. (c) SLSC beamformed photoacoustic image overlaid on US image. The photoacoustic images were acquired with $40.5 \mathrm{~mJ}$ energy. (d) Color Doppler confirmation of blood flow in the hepatic vein. 


\subsection{In Vivo Liver Imaging}

Figure 7(a) shows the US image of an in vivo hepatic vein and a coregistered photoacoustic image created with DAS beamforming overlaid on this US image. The photoacoustic SLSC image created with the same channel data is shown in Fig. 7(b). This SLSC image is overlaid on the US image in Fig. 7(c). The color Doppler image confirming the location of blood vessels in the liver is shown in Fig. 7(d). Similar to the pancreas image, the blood signals in this photoacoustic image of the liver do not encompass the entire vessel location.

Figure 8 shows an US image of the hepatic vein, which was confirmed with Doppler imaging. The diameter of the vein in Fig. 8 is $\sim 3 \mathrm{~mm}$, which is smaller than the diameter of the vessels shown in Figs. 5 and 7. When imaging these smaller-diameter vessels, we observed a change in photoacoustic signal appearance based on the location of the light source. In particular, when light from the fiber bundle was directed at this vein, we saw a significantly more focused photoacoustic signal. In contrast, when the light source was purposely directed away from this vein, the signal was more diffuse. This change in signal appearance is also evident in Video 1, and it is likely due to laser excitation of the blood inside of the hepatic vein (producing more focused signals) or laser excitation of the surrounding tissue (producing more diffuse signals).
Although the appearance of focused versus diffuse signals were highly repeatable in real time, it is understandable that the more diffuse signals associated with the tissue can be mistaken for photoacoustic signal artifacts and can also confuse the reading of the still images. Therefore, one option to mitigate this confusion is to reduce the dynamic range of the photoacoustic images, as shown in Fig. 8 (bottom). This reduction is sufficient because the diffuse signals have consistently lower amplitude than the focused signals. However, the distinction between the two types of signals is more obvious with the higher dynamic range shown in Fig. 8 (top).

Figure 9(a) shows additional examples of the focused photoacoustic signal from the hepatic vein acquired at multiple energy levels. Each image is displayed with a dynamic range that was chosen to optimize signal visibility. The focused signal was not present at energies of 20 and $25 \mathrm{~mJ}$, even with the higher dynamic range values. Beyond $25 \mathrm{~mJ}$, the focused nature of the signals in the vessels was more apparent, particularly with reduced dynamic range values.

Figure 9(b) summarizes contrast measurements of the focused signals shown in Fig. 9(a) and diffuse signals acquired with the same energy levels. Although the diffuse signals at each energy level are not shown, they look similar to the examples shown in Fig. 8 and in Video 1. The contrast measurements are independent of the displayed dynamic range, so they can be relied upon as objective measurements of signal visibility.

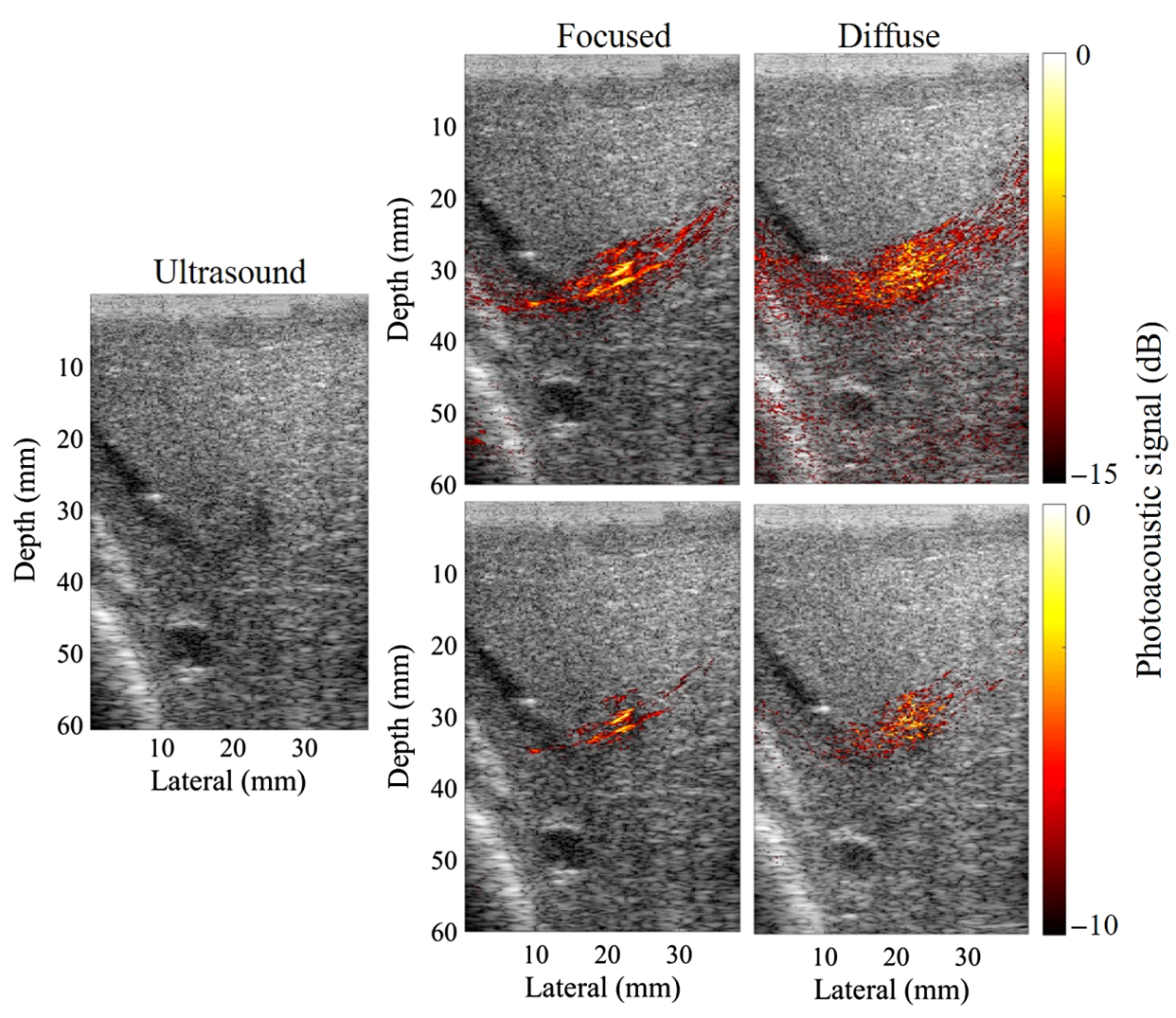

Fig. 8 US image of the hepatic vein and corresponding DAS photoacoustic images (overlaid on US image), demonstrating focused and diffuse signals acquired with a laser energy of $30 \mathrm{~mJ}$. The photoacoustic images are displayed with dynamic ranges of $15 \mathrm{~dB}$ (top) and $10 \mathrm{~dB}$ (bottom), as noted by the maximum and minimum values on the color bars. Reducing the dynamic range emphasizes the appearance of the focused signal and limits the appearance of the diffuse signal. More examples of focused and diffuse signals from this image acquisition sequence are shown in Video 1 (Video 1, MPEG, 2.8 MB [URL: https://doi.org/10.1117/1.JBO.24.12.121905.1]). 
(a)

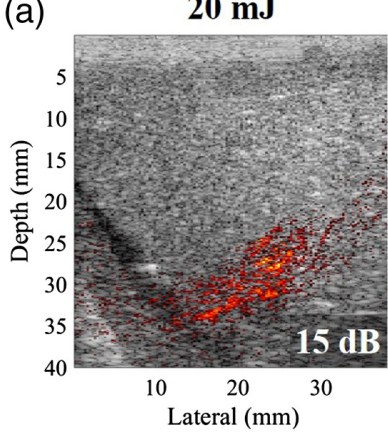

$25 \mathrm{~mJ}$

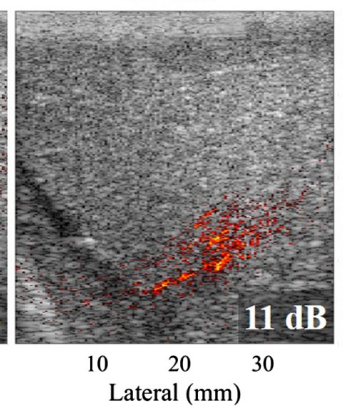

$30 \mathrm{~mJ}$

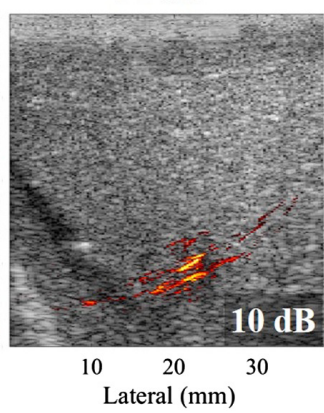

$35 \mathrm{~mJ}$

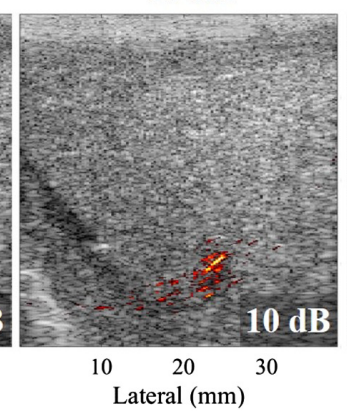

$40 \mathrm{~mJ}$

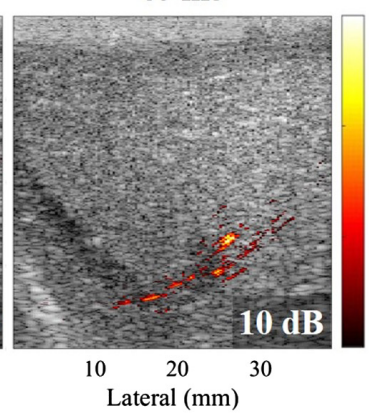

(b)

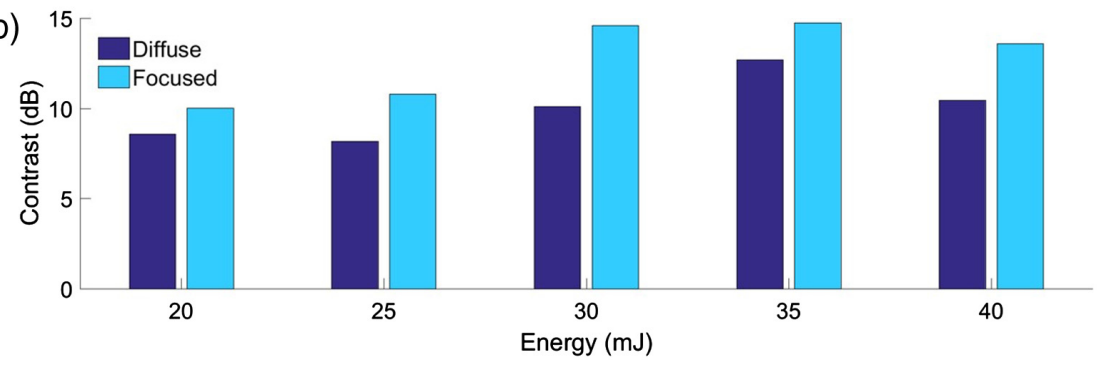

Fig. 9 (a) Focused photoacoustic signals in the hepatic vein, acquired with varying laser energy. Each image is annotated with the dynamic range chosen to optimize the signal display. (b) Measured contrast of focused and diffuse signals at the various laser energies.

At each energy level, the focused signal has consistently higher contrast when compared to the diffuse signals, which further supports the dynamic range reduction in Figs. 8 and 9(a), in order to emphasize the appearance of focused signals and limit the appearance of diffuse signals.

The focused and diffuse appearance of the photoacoustic signals observed in the liver is likely caused by the blood in the liver, particularly when considering that the prominent diffuse appearance was not observed in the pancreas, which generally has lower optical absorption and scattering than the liver (see Table 1).

\subsection{Three-Dimensional Vessel Imaging}

Figure 10 shows multiple display formats for the 3-D vessel image data that we acquired with robotic assistance (as described in Sec. 2.2). The left column of Fig. 10 displays results associated with the photoacoustic signal, while the right column displays results associated with the color Doppler signal, which was used for confirmation of blood flow. Figure 10(a) shows the 3-D stack of images used for forming the volumetric data. Biplanar views of intersecting elevational and lateral slices are shown in Fig. 10(b), with the chosen slices outlined in green in Fig. 10(a).

An isosurface of the segmented vessels based on the photoacoustic and Doppler volumetric image data is displayed in Fig. 10(c). There are size and shape differences between these two segmented vessels that occur because the photoacoustic signal is only present near the location of the 5 -mm-diameter light source, which is unable to simultaneously illuminate all regions containing the blood signals. This capability to confidently limit the field of view (FOV) to a specific ROI at the surgical site is one of the main advantages of using photoacoustic imaging to guide surgeries. The presence of a major blood vessel near the surgical ROI can be highlighted, rather than displaying multiple regions with blood flow through the use of Doppler imaging. Although limiting the FOV may be possible with Doppler imaging, doing so would require coordination of identifying tool tips and possible adjustment of knobs on the US scanner to manually limit the FOV.

Figure 10(d) shows the two segmented vessels of Fig. 10(c) overlaid in the same spatial coordinate system. In addition to the smaller FOV with photoacoustic images (and hence the smaller vessel volume), differences between the two segmented vessels also exist because of the differences in the image acquisition procedure. Photoacoustic images were acquired as the robot stepped forward across the liver surface, while Doppler images were acquired as the robot retraced its steps in the opposite direction. Considering these two different motion directions, it is highly possible that the tissue deformed differently during the acquisition of each volume, which would cause the vessels to be shifted relative to each other.

\subsection{Histopathology}

Figure 11 shows the histology results for the liver and pancreatic tissues after applying the multiple energies over the time duration shown in Fig. 1. No pathology was observed in the preexperiment control in either the pancreas or the liver. There was no necrosis observed in the pancreas after being exposed to the laser. However, Fig. 11(a) shows an example of the mild degeneration in the post-experiment control and lasered pancreatic tissue. This mild cell degeneration observed in the pancreas after imaging was likely caused by the pressure of the US probe and other tissue handling. In the liver, necrosis was observed in both the lasered tissue and the post-experiment control tissue, as demonstrated in Fig. 11(b). There was also hemorrhaging and inflammation present in the lasered section of liver tissue. We interpret these findings within the scope of our overall study design in Sec. 4. 

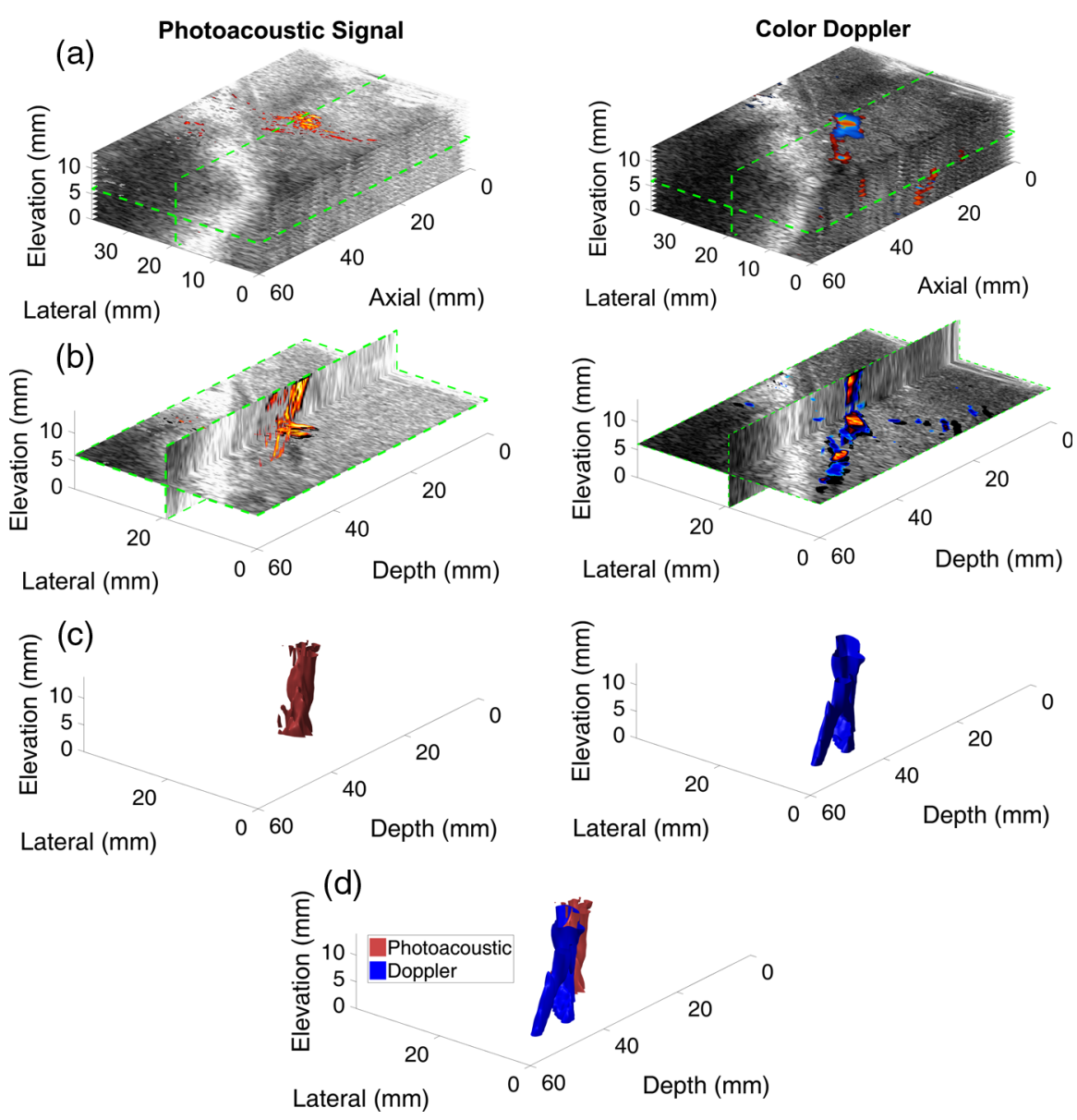

Fig. 10 The 3-D representations of the hepatic vein created from DAS photoacoustic and color Doppler images. (a) Volume stack of photoacoustic and Doppler images acquired with a robot translating in 1-mm increments. (b) Biplanar cross sections through the volume stack. (c) 3-D reconstructions of the hepatic vein segmented from photoacoustic and Doppler images. (d) Segmented vessels from photoacoustic and Doppler images combined to show similarities between the two segmented vessel structures.

(a)

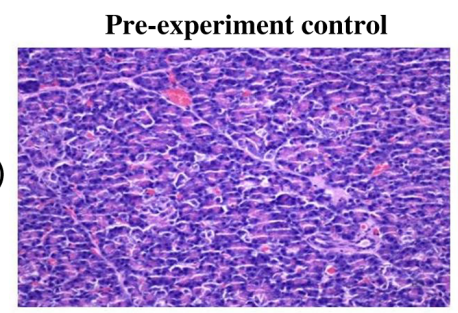

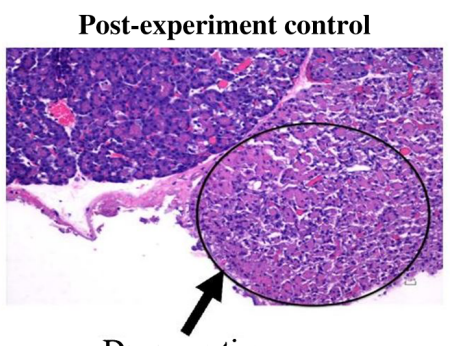

Degeneration
Post-experiment lasered region

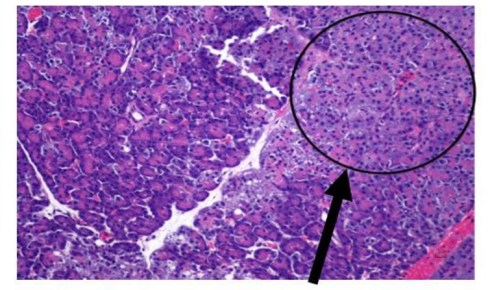

Degeneration

(b)
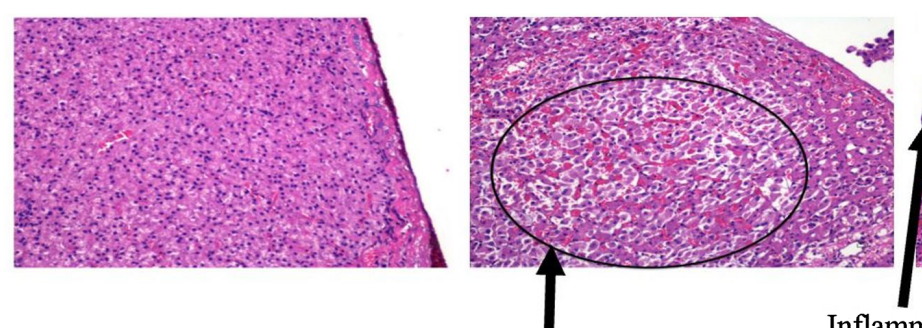

Degeneration/necrosis

Inflammation

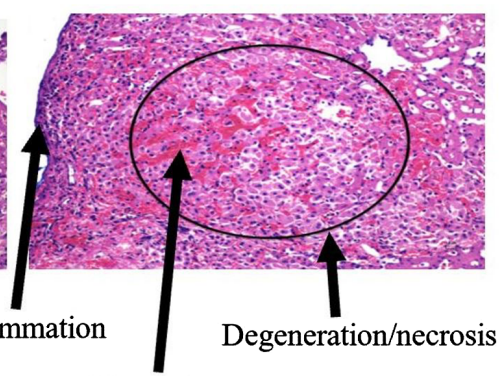

Hemorrhage

Fig. 11 Histopathology results of (left) pre-experiment control, (middle) post-experiment control, and (right) post-experiment lasered tissue for the (a) pancreas and (b) liver at 200x magnification. 


\section{Discussion}

We successfully visualized in vivo blood vessels surrounding the pancreas and within the liver. This success has two immediate implications for photoacoustic-guided abdominal surgeries. First, the visualization of major blood vessels during surgery can possibly help surgeons to navigate around these vessels during tissue resection procedures. Second, this approach can be used to assist with estimating and targeting areas where cauterization of the major blood vessels is necessary in order to reduce significant bleeding and blood loss during surgery. The proposed technique can enable these benefits by adjusting the location of the light source to determine the location of a major hepatic blood vessel based on its appearance as a focused signal, rather than a diffuse signal (which tends to indicate the presence of predominantly liver tissue). This diffuse signal in liver tissue is likely caused by the presence of multiple small blood vessels within liver tissue that are either located in the image plane or located off-axis from the image plane. This interpretation is particularly convincing when considering that the diffuse signals observed in vivo were not present during the ex vivo liver experiments. One example of the benefits of this photoacoustic imaging technique over standard color Doppler imaging methods is presented and described in Sec. 3.4.

Although this study was performed to investigate feasibility in the context of abdominal surgeries, there are a range of additional interventional procedures that might benefit from these findings, such as photoacoustic imaging of radiofrequency ablation lesions. For example, when detecting these lesions in the liver using photoacoustic imaging, the surrounding tissue is expected to appear as diffuse signals, while the blood contained in major vessels will likely appear as more focused signals.

The distinction between focused and diffuse signals does not seem to translate to vessels that appear as circular in the US images with diameters as large as $1 \mathrm{~cm}$. Instead of visualizing all blood content in the photoacoustic images of these larger vessels, only the center of these vessels are apparent, as shown in the pancreas and liver images of Figs. 5 and 7, respectively. Visualizing only the center of the vessels is not ideal when attempting to identify the location of vessel boundaries. This appearance of the vessel center seems unique to the in vivo imaging cases and is not observed in the ex vivo results (e.g., Fig. 6), where only the boundaries of the vessels are apparent. Therefore, these in vivo results for the larger vessels possibly reveal some underlying physical property of these vessels that is not present in the smaller vessels. This limited view of the contents within the vessel can also be caused by the limited size of the light source (i.e., 5-mm diameter) relative to the $1-\mathrm{cm}$ diameter of these vessels (although intuition suggests that light scattering and diffusion should help to spread the light more evenly throughout the vessel).

While results are generally promising, we identified two additional limitations that require extensive consideration prior to and/or during future clinical implementation of the proposed technique. The first challenge is particularly familiar to photoacoustic imaging-i.e., the presence of artifacts. We investigated the various signal sources with ex vivo experiments and coherence-based SLSC beamforming, with results supporting our hypotheses that the unexpected signals appearing in vivo (e.g., Fig. 5) were likely caused by blood vessels outside of the imaging plane. This is an important finding for future in vivo vessel imaging experiments, particularly when the light source is stationary relative to a nonstationary organ that is subjected to respiratory motion. To overcome this limitation, the 3-D structure of potential out-of-plane vessels can be confirmed with volumetric imaging, as illustrated in Fig. 10.

The second limitation is that the fluence levels needed to visualize the major blood vessels in the liver exceed the safety limit for skin (i.e., $25.2 \mathrm{~mJ} / \mathrm{cm}^{2}$ when using a 750-nm wavelength laser). ${ }^{58}$ Specifically, an energy of $30 \mathrm{~mJ}$ was required to visualize the hepatic vein with $15.5 \mathrm{~dB}$ of contrast, as shown in Fig. 8, and this energy translates to a fluence of $153 \mathrm{~mJ} / \mathrm{cm}^{2}$, based on the calculations described in Sec. 2.3. However, these fluence calculations are conservative (as noted in Sec. 2.3). In addition, tissues from other organs, such as the liver, may have a higher safety threshold, considering the significantly different optical and thermal properties noted in Table 1.

The histopathological analysis conducted on resected tissues after implementing photoacoustic imaging revealed that there was no significant laser-related damage to the pancreatic tissue (defined as cell necrosis). On the other hand, cell necrosis was observed in the liver tissues after laser application. We attribute the differences in laser-related damage to these two organs to the longer laser exposure times for the liver experiment (see Fig. 3) and to the higher optical scattering in the liver (see Table 1). Long-term studies are needed to determine if cell degeneration in pancreatic tissue and cell necrosis in liver tissue will be chronic issues for patients that might otherwise benefit from the proposed photoacoustic imaging procedure. Another point to consider is that the liver tissue is highly regenerative and these regenerative properties can possibly enable the application of $\geq 30 \mathrm{~mJ}\left(153 \mathrm{~mJ} / \mathrm{cm}^{2}\right)$ of optical energy without significant long-term, laser-related damage. This level of energy can also be applied with a significantly lower fluence if the area of illumination is increased, which may be safer for the liver tissue.

Using Eq. (1), we calculated that a surface area of $1.19 \mathrm{~cm}^{2}$ (which corresponds to a diameter of $1.23 \mathrm{~cm}$, assuming a circular illumination profile) is needed in order to utilize $30 \mathrm{~mJ}$ of energy within the $25.2 \mathrm{~mJ} / \mathrm{cm}^{2}$ fluence safety limits that are currently defined for skin. ${ }^{58}$ Therefore, it seems feasible to design a light delivery system that delivers this area profile either on the tissue surface (as performed during our experiments) or when inserted inside the liver (particularly in cases such as biopsies, radiofrequency ablations, or other procedures that require tool insertion within tissue). Characterization of the specific safety limits for liver tissue would also be helpful, and this will be the focus of our future work.

Future work will also explore the illumination of the pancreas and liver tissue with a light source that would enable differentiation of diffuse from focused signals without requiring motion of the light source. This can be accomplished by increasing the area of the illumination. In addition to decreasing the incident fluence needed to deliver a suitable energy to visualize the abdominal vasculature, as described above, this larger light source will also provide a slightly wider FOV for surgeons to visualize a larger portion of the vasculature relative to the location of a surgical tool.

\section{Conclusion}

This work is the first to demonstrate in vivo blood vessel visualization with possible applications to a range of photoacousticguided pancreatic and liver surgeries. We observed a change in the photoacoustic signal appearance when the light source encountered a major vessel in the liver. These findings are promising for surgical guidance when the probe is fixed and the light 
source is used to interrogate the surgical workspace. While the applied energies revealed no laser-related damage (i.e., cell necrosis) to pancreatic tissues, additional work is required to determine tissue-specific energy safety limits for liver tissue to further assess the feasibility of photoacoustic-guided liver surgery. This is particularly true when considering that a minimum of $30 \mathrm{~mJ}$ of laser energy was required to confidently visualize the focused photoacoustic signals that are characteristic of major vessels in the in vivo liver.

\section{Disclosures}

The authors have no relevant financial interests in this paper and no potential conflicts of interest to disclose.

\section{Acknowledgments}

This work was supported by NSF CAREER Award \#1751522, REU Supplement to NSF CAREER Award \#1751522, and the NSF Computational Sensing and Medical Robotics Research Experience for Undergraduates program at Johns Hopkins University (Award \#1460674). The authors thank Nicholas Louloudis, Sue Eller, Ivan George, Dr. Lingdi Yin, and Dr. Liang Wang for animal care and surgery support; Patricia Wilcox for assistance with histopathology preparation; and the Carnegie Center for Surgical Innovation. They also acknowledge the support of NVIDIA Corporation with the donation of the Titan Xp GPU that was used for portions of the signal processing required to display images presented in this paper.

\section{References}

1. S. J. McNally et al., "Factors in perioperative care that determine blood loss in liver surgery," $H P B$ 14, 236-241 (2012).

2. E. M. Alkozai, T. Lisman, and R. J. Porte, "Bleeding in liver surgery: prevention and treatment," Clin. Liver Dis. 13, 145-154 (2009).

3. F. Romano et al., "Bleeding in hepatic surgery: sorting through methods to prevent it," HPB Surg. 2012, 1-12 (2012).

4. A. Lakshmanan et al., "Bleeding complication after pancreatic surgery: role of harmonic scalpel," Indian J. Surg. 75(Suppl. 1), 436-438 (2013).

5. G. Rajarathinam et al., "Post pancreaticoduodenectomy haemorrhage: outcome prediction based on new ISGPS clinical severity grading," $H P B$ 10(5), 363-370 (2008).

6. M. N. Wente et al., "Postpancreatectomy hemorrhage (PPH): an International Study Group of Pancreatic Surgery (ISGPS) definition," Surgery 142, 20-25 (2007).

7. M. S. Taljanovic et al., "Artifacts in musculoskeletal ultrasonography," Semin. Musculoskelet Radiol. 18, 3-11 (2014).

8. J. Huang et al., "Imaging artifacts of medical instruments in ultrasoundguided interventions," Technical Report (2007).

9. M. A. Lediju et al., "Quantitative assessment of the magnitude, impact and spatial extent of ultrasonic clutter," Ultrason. Imaging 30(3), 151-168 (2008).

10. M. J. Modica, K. M. Kanal, and M. L. Gunn, "The obese emergency patient: imaging challenges and solutions," RadioGraphics 31, 811-823 (2011).

11. M. A. L. Bell and J. Shubert, "Photoacoustic-based visual servoing of a needle tip," Sci. Rep. 8(1), 15519 (2018).

12. T. R. Beard, D. L. Kaserman, and R. Osterkamp, The Global Organ Shortage: Economic Causes, Human Consequences, Policy Responses, The Independent Review, The Independent Institute (2013).

13. R. Bouchard, O. Sahin, and S. Emelianov, "Ultrasound-guided photoacoustic imaging: current state and future development," IEEE Trans. Ultrason. Ferroelectr. Freq. Control 61, 450-466 (2014).

14. M. Xu and L. V. Wang, "Photoacoustic imaging in biomedicine," Rev. Sci. Instrum. 77, 041101 (2006).

15. L. G. Montilla et al., "Real-time photoacoustic and ultrasound imaging: a simple solution for clinical ultrasound systems with linear arrays," Phys. Med. Biol. 58, N1-N12 (2013).
16. X. Wang et al., "Photoacoustic imaging with a commercial ultrasound system and a custom probe," Ultrasound Med. Biol. 37(3), 484-492 (2011).

17. X. Wang et al., "Three-dimensional laser-induced photoacoustic tomography of mouse brain with the skin and skull intact," Opt. Lett. 28, 1739-1741 (2003).

18. L. G. Schulz, "The optical constants of silver, gold, copper, and aluminum I the absorption coefficient k," J. Opt. Soc. Am. 44, 357-362 (1954).

19. B. Eddins and M. A. L. Bell, "Design of a multifiber light delivery system for photoacoustic-guided surgery," J. Biomed. Opt. 22(4), 041011 (2017).

20. M. A. L. Bell et al., "Localization of transcranial targets for photoacoustic-guided endonasal surgeries," Photoacoustics 3(2), 78-87 (2015).

21. M. Allard, J. Shubert, and M. A. L. Bell, "Feasibility of photoacousticguided teleoperated hysterectomies," J. Med. Imaging 5(2), 021213 (2018).

22. M. Allard, J. Shubert, and M. A. L. Bell, "Technical note: feasibility of photoacoustic guided hysterectomies with the da Vinci robot," Proc. SPIE 10576, 105760A (2018).

23. J. Shubert and M. A. L. Bell, "Photoacoustic imaging of a human vertebra: implications for guiding spinal fusion surgeries," Phys. Med. Biol. 63(14), 144001 (2018).

24. W. Xia et al., "Interventional photoacoustic imaging of the human placenta with ultrasonic tracking for minimally invasive fetal surgeries," Lect. Notes Comput. Sci. 9349, 371-378 (2015).

25. N. Gandhi et al., "Photoacoustic-based approach to surgical guidance performed with and without a da Vinci robot," J. Biomed. Opt. 22(12), 121606 (2017).

26. S. Kim et al., "Feasibility of photoacoustic image guidance for telerobotic endonasal transsphenoidal surgery," in 6th IEEE Int. Conf. Biomed. Rob. and Biomechatron., IEEE, pp. 482-488 (2016).

27. S. Kim et al., "Improving the safety of telerobotic drilling of the skull base via photoacoustic sensing of the carotid arteries," in IEEE Int. Conf. Rob. and Autom., pp. 2385-2390 (2017).

28. H. Moradi, S. Tang, and S. E. Salcudean, "Towards intra-operative prostate photoacoustic imaging: configuration evaluation and implementation using the da Vinci research kit," IEEE Trans. Med. Imaging 38, 57-68 (2019).

29. J. Shubert and M. A. L. Bell, "Photoacoustic based visual servoing of needle tips to improve biopsy on obese patients," in IEEE Int. Ultrason. Symp., pp. 1-4 (2017).

30. E. R. Hill et al., "Interventional multi-spectral photoacoustic imaging in laparoscopic surgery," Proc. SPIE 9708, 97080B (2016).

31. T. Mitcham et al., "Photoacoustic imaging driven by an interstitial irradiation source," Photoacoustics 3(2), 45-54 (2015).

32. N. Benvenuto et al., "Real-time monitoring of tumor-specific indocyanine green hepatic photothermal ablation using photoacoustic imaging," J. Vasc. Interventional Radiol. 28(2), S102-S103 (2017).

33. C.-T. Germer et al., "Optical properties of native and coagulated human liver tissue and liver metastases in the near infrared range," Technical Report (1998).

34. M. A. L. Bell et al., "Short-lag spatial coherence beamforming of photoacoustic images for enhanced visualization of prostate brachytherapy seeds," Biomed. Opt. Express 4(10), 1964-1977 (2013).

35. E. V. Salomatina et al., "Optical properties of normal and cancerous human skin in the visible and near-infrared spectral range," J. Biomed. Opt. 11(6), 064026 (2006).

36. S. Jiang et al., "Effects of thermal properties and geometrical dimensions on skin burn injuries," Burns 28(8), 713-717 (2002).

37. J.-P. Ritz et al., "Optical properties of native and coagulated porcine liver tissue between 400 and 2400 nm," Lasers Surg. Med. 29(3), 205-212 (2001).

38. J.-P. Ritz et al., "Comparison of the optical properties of human liver tissue and liver metastases and investigation of the dynamic temperature behavior of optical tissue properties," Proc. SPIE 2970, 500-507 (1997).

39. J. Valvano, J. Cochran, and K. Diller, "Thermal conductivity and diffusivity of biomaterials measured with self-heated thermistors," Int. J. Thermophys. 6(3), 301-311 (1985).

40. F. A. Duck, Physical Properties of Tissues: A Comprehensive Reference Book, Academic Press, London (2013).

41. P. Hasgall et al., "IT'IS database for thermal and electromagnetic parameters of biological tissues," 4 (2018). 
42. J. MacLeod, D. Blanc, and M. Colles, "Measurement of the opticalabsorption coefficient at 1.06-mum of various tissues using the photoacoustic effect," Lasers Surg. Med. 8(2), 143-143 (1988).

43. M. J. van Gemert et al., "Optical properties of human blood vessel wall and plaque," Lasers Surg. Med. 5(3), 235-237 (1985).

44. P. Saccomandi et al., "Theoretical analysis and experimental evaluation of laser-induced interstitial thermotherapy in ex vivo porcine pancreas," IEEE Trans. Biomed. Eng. 59(10), 2958-2964 (2012).

45. M. M. Swindle et al., "Swine as models in biomedical research and toxicology testing," Vet. Pathol. 49, 344-356 (2011).

46. Z. Yifei et al., "Comparison of morphology and microstructural components of hepatic portal vein between human and pig," J. Huazhong Univ. Sci. Technol. 25(4), 419-422 (2005).

47. K.-T. von Trotha et al., "Vascular anatomy of the small intestine: a comparative anatomic study on humans and pigs," Int. J. Colorectal Dis. 30, 683-690 (2015).

48. J. Ferrer et al., "Pig pancreas anatomy: implications for pancreas procurement, preservation, and islet isolation," Transplantation $\mathbf{8 6}$, 1503-1510 (2008).

49. J. M. Kim and H. W. Lee, "Anatomy of the pig," in 8th Int. Single Top. Symp. Korean Assoc. HBP Surg., pp. 129-132 (2013).

50. K. M. Kempski et al., "In vivo demonstration of photoacoustic-guided liver surgery," Proc. SPIE 10878, 108782T (2019).

51. J. Shubert and M. A. L. Bell, "A novel drill design for photoacoustic guided surgeries," Proc. SPIE 10494, 104940J (2018).

52. K. Sivasubramanian et al., "Optimising probe holder design for sentinel lymph node imaging using clinical photoacoustic system with Monte Carlo simulation," Proc. SPIE 10064, 100645N (2017).

53. K. Sivasubramanian et al., "Optimizing light delivery through fiber bundle in photoacoustic imaging with clinical ultrasound system: Monte Carlo simulation and experimental validation," J. Biomed. Opt. 22(4), 041008 (2016).

54. G. S. Sangha, N. J. Hale, and C. J. Goergen, "Adjustable photoacoustic tomography probe improves light delivery and image quality," Photoacoustics 12, 6-13 (2018).

55. V. Periyasamy and M. Pramanik, "Monte Carlo simulation of light transport in tissue for optimizing light delivery in photoacoustic imaging of the sentinel lymph node," J. Biomed. Opt. 18(10), 106008 (2013).

56. Adapted from https://www.biodigital.com.

57. M. A. Lediju et al., "Short-lag spatial coherence of backscattered echoes: imaging characteristics," IEEE Trans. Ultrason. Ferroelectr. Freq. Control 58, 1377-1388 (2011).

58. Laser Institute of America, "American National Standards for safe use of lasers," ANSI Z136.1 (2014).

Kelley M. Kempski received her bachelor's degree in biomedical engineering from the University of Delaware in 2018. She completed the NSF Research Experience for Undergraduates program in Photoacoustic and Ultrasonic Systems Engineering (PULSE) Lab at Johns Hopkins University during the summer of 2018, where she conducted research in photoacoustic imaging of the pancreas and liver. She will commence graduate studies in biomedical engineering at Johns Hopkins University. She is a recipient of the NSF graduate research fellowship, and her research interests include photoacoustic imaging, translational research, and ultrasound imaging.

Alycen Wiacek is a graduate student of electrical and computer engineering (ECE) at Johns Hopkins University. She received her BS degree in ECE from Wayne State University in 2014, her MS degree in ECE from Oakland University in 2015, and her MSE degree in electrical engineering from Johns Hopkins University in 2019. Her research interests include coherence-based beamforming, breast ultrasound, photoacoustic imaging, and translational research.

Michelle Graham is a graduate student of ECE at Johns Hopkins University. She received her BS degree in biophysics (engineering minor) from the University of Scranton in 2015 and her MSE degree in electrical engineering from Johns Hopkins University in 2018. She is a recipient of the NSF graduate research fellowship, and her research interests include intraoperative photoacoustic imaging, neurosurgical guidance, and translational research.
Eduardo González is a graduate student of biomedical engineering at Johns Hopkins University. He received his BS degree in electronic engineering from the Pontificia Universidad Catolica del Peru (PUCP) in 2013, and his master's degree in mechatronic engineering from PUCP in 2016. He is a recipient of the Fulbright Fellowship and his research interests include translational research, real-time ultrasound imaging, advanced beamforming, and quantitative ultrasound.

Bria Goodson is an undergraduate student majoring in biology at Delta State University. She completed the McNair Scholar Research Experience for Undergraduates program in the PULSE Lab at Johns Hopkins University during the summer of 2018 , where she conducted research on laser tissue interactions during photoacoustic imaging of the pancreas and liver.

Derek Allman received his BS degree in electrical engineering from the Ohio State University in 2016. He completed his MSE degree in electrical engineering at Johns Hopkins University in 2019, and his research interests include machine learning, signal processing, medical imaging, and data analysis.

Jasmin Palmer received her BS degree in mechanical engineering from Massachusetts Institute of Technology in 2019. She completed the Leadership Alliance Research Experience for Undergraduates program in the PULSE Lab at Johns Hopkins University during the summer of 2018, where she conducted research related to custom light delivery systems for robotic photoacoustic imaging. She will commence graduate studies in mechanical engineering at Stanford University.

Huayu Hou is a graduate student in ECE at Johns Hopkins University. He received his BS degree in electrical engineering from Wuhan University in 2017. He completed his MSE degree in electrical engineering at Johns Hopkins University in 2019, and his research interests include medical imaging, statistical modeling, and optics.

Sarah Beck is a board-certified veterinary anatomic pathologist and an assistant professor of molecular and comparative pathobiology at Johns Hopkins University School of Medicine. She received her BS degree in marine biology from the University of Maryland in 2002, her DVM from Virginia-Maryland Regional College of Veterinary Medicine in 2007, and her PhD in cellular and molecular medicine from Johns Hopkins University in 2014. She completed her comparative anatomic pathology residency in the Department of Molecular and Comparative Pathobiology at Johns Hopkins University. Her research interests include studying CD8 T cell-mediated control of lentiviral infections using animal models.

Jin He is an assistant professor of surgery at Johns Hopkins University School of Medicine. He is a surgical oncologist specializing in tumors from the hepato-pancreato-biliary organs. He received his medical degree from Beijing Medical University in 1996 and a PhD in oncology from Fudan University Shanghai Medical College in 2001. He completed the Halsted general surgery residency training at Johns Hopkins, followed by an ACGME-accredited fellowship in complex general surgical oncology at Johns Hopkins in 2015. His research focuses on personalized treatment through stratifying pancreatic tumors on their genetic features. He serves on several national committees, including the NCCN panel on neuroendocrine tumors and the Alliance for Clinical Trials in Oncology.

Muyinatu A. Lediju Bell is an assistant professor of ECE, biomedical engineering, and computer science at Johns Hopkins University, where she founded and directs the PULSE Lab. She received her $\mathrm{PhD}$ in biomedical engineering from Duke University in 2012, completed research abroad as a Whitaker International fellow in 2010, and earned her BS degree in mechanical engineering (biomedical engineering minor) from Massachusetts Institute of Technology in 2006. Her research interests revolve around ultrasound and photoacoustic imaging, photoacoustic-guided surgery, robot-assisted imaging, and the design of innovative medical imaging technologies that will improve the standard of cancer patient care. 